\title{
ARTICLE \\ $\alpha 7$ Nicotinic receptor-modulating agents reverse the hyperdopaminergic tone in the MAM model of schizophrenia
}

\author{
Gilda A Neves (iD) and Anthony A Grace (iD)
}

\begin{abstract}
Recent evidence has emerged supporting a role for the cholinergic system in schizophrenia, including the potential of a7 modulators as a treatment strategy. However, preclinical studies to date have relied on studies in normal systems rather than on a validated developmental model of schizophrenia. Furthermore, there have been only few studies on whether orthosteric and allosteric modulators have differential impacts in such models. Thus, we investigated the effects of a7 agonists and positive allosteric modulators (PAMs) on dopamine (DA) neuron activity in the ventral tegmental area (VTA) in the methylazoxymethanol acetate (MAM) developmental disruption model of schizophrenia. Four different drugs were evaluated: PNU282987 (full agonist), SSR180711 (partial agonist) NS1738 (PAM type I) and PNU120596 (PAM type II). PNU120596 increased the number of spontaneously active VTA DA neurons in normal rats. In contrast, PNU282987 and SSR180711 reduced the hyperdopaminergic tone in MAM rats. This appeared to be due to effects on DA afferent regulation, in that PNU282987 or SSR180711 infusion into the ventral hippocampus of MAM rats replicated the decrease in the number of spontaneously active VTA DA neurons. In contrast, infusion of the same drugs into the basolateral amygdala increased the number of spontaneously active VTA DA neurons in normal rats without impacting MAM rats. These data suggest that a7 receptors may represent a promising target in the development of new pharmacological therapies for schizophrenia.
\end{abstract}

Neuropsychopharmacology (2018) 43:1712-1720; https://doi.org/10.1038/s41386-018-0066-0

\section{INTRODUCTION}

Schizophrenia is comprised of several symptom classes that likely involve distinct brain systems. Most investigations into schizophrenia have revolved around the dopaminergic (DA) and glutamatergic systems, whereas the cholinergic system has received little attention. Indeed, elevated choline levels [1] and alterations in cholinergic neurons in the ventral striatum and pendunculopontine tegumentum [2] have been reported in schizophrenia, suggesting that an imbalance of cholinergic neurotransmission could underlie the disorder. Furthermore, epidemiological data demonstrate a high prevalence (80-90\%) of tobacco smoking among schizophrenia patients [3], which are considered "heavy" nicotine users, experience greater reinforcing and more severe withdraw symptoms than non-psychotic smokers [4]. One possible explanation is the self-medication hypothesis, supported by the procognitive effects of nicotine in smoking and non-smoking schizophrenia patients [5]. These findings point to disfunctions in the nicotinic system in schizophrenia pathophysiology.

There is a growing interest in the study of a7 nicotinic acetylcholine receptors (nAChR) in schizophrenia symptomatology and as a possible treatment target. a7 $\mathrm{nAChR}$ are expressed in several brain areas involved in the pathophysiology of schizophrenia including the hippocampus (Hipp), ventral tegmental area (VTA) and striatum. These homomeric receptors present an extremely fast activation/desensitization [6] and differ from other nAChRs due to their high $\mathrm{Ca}^{2+}$ permeability [7] and frequent expression in pre-synaptic terminals $[8,9]$, defining a role in controlling the release of several key neurotransmitters. Furthermore, these receptors are involved in several factors disrupted in schizophrenia, such as sensory gating, attention, memory and cognitive flexibility $[10,11]$. Post-mortem studies report a decrease in a7 subunit protein and mRNA levels in the frontal and cingulate cortex, Hipp and thalamus of schizophrenia patients [12, 13]. Data support a genetic link between schizophrenia symptoms and chromosome 15 polymorphisms located in the a7 subunit gene $[14,15]$. Although the role of $a 7 n A C h R$ in the pathophysiology of schizophrenia is not fully understood, the potential of a7 modulators as an alternative treatment strategy is under investigation [11].

Novel compounds that selectively bind to a7 nAChR with a broad range of intrinsic activities have been developed. Like other ligand-gated ion channels, a7 nAChR present several allosteric binding sites. Thus, beside the classic agonists, two main classes of positive allosteric modulators (PAMs) have been identified. The type I PAMs act by increasing the amplitude of the ionic current induced by acetylcholine in vitro with minimum change in receptor desensitization kinetics [16]. In contrast, the type II PAMs increase agonist-induced peak current while substantially slowing receptor desensitization [17]. Although the molecular mechanism of action of these drugs is well characterized, whether these differences result in distinct in vivo effects is unclear.

Alpha7 nAChR agonists and PAMs therapeutic potential investigation have focused on cognitive remediation [18] and studies to date have been restricted to normal systems rather than on well-validated models of schizophrenia. The

${ }^{1}$ Departments of Neuroscience, Psychiatry and Psychology, University of Pittsburgh, Pittsburgh, PA, USA

Correspondence: Anthony A Grace (graceaa@pitt.edu)

Received: 22 December 2017 Accepted: 2 April 2018

Published online: 19 April 2018 
Table 1. $\alpha 7 \mathrm{nAChR}$ ligands, molecular mechanism of action and doses

\begin{tabular}{|c|c|c|c|c|c|}
\hline \multirow[t]{2}{*}{ Drug } & \multirow[t]{2}{*}{$\begin{array}{l}\text { Mechanism of } \\
\text { Action }\end{array}$} & \multicolumn{2}{|c|}{$\begin{array}{l}\text { Systemic } \\
\text { administration (i.v.) }\end{array}$} & \multicolumn{2}{|c|}{$\begin{array}{l}\text { Intracerebral } \\
\text { administration }\end{array}$} \\
\hline & & Dose & Reference & Dose & Reference \\
\hline PNU282987 & Full agonist & $1 \mathrm{mg} / \mathrm{kg}$ & [51] & $2.5 \mu \mathrm{g}$ & {$[52]$} \\
\hline SSR180711 & Partial agonist & $3 \mathrm{mg} / \mathrm{kg}$ & [53] & $5.0 \mu \mathrm{g}$ & [54] \\
\hline NS1738 & PAM type I & $0.1 \mathrm{mg} / \mathrm{kg}$ & {$[40]$} & & \\
\hline PNU120596 & PAM type II & $1 \mathrm{mg} / \mathrm{kg}$ & [17] & & \\
\hline
\end{tabular}

methylazoxymethanol acetate (MAM) development disruption model is considered among the most translational models of schizophrenia [19], characterized by a disruption in the Hippstriatum-VTA circuit that leads to a hyper-DA state driven by an increase in the number of DA neurons spontaneously active [20]. We now show that a7 nAChR agonists and PAMs can affect midbrain DA neurotransmission in vivo in a state-dependent manner that may have therapeutic implications.

\section{MATERIALS AND METHODS \\ Drugs}

MAM was obtained from Midwest Research Institute (Kansas City, Missouri). Chloral hydrate, PNU282987 hydrate and PNU120596 were purchased from Sigma-Aldrich, and SSR180711 hydrochloride and NS1738 from Tocris Bioscience. For systemic administration, MAM, chloral hydrate, PNU282987 and SSR180711 were dissolved in saline $(0.9 \% \mathrm{NaCl})$. PNU120596 and NS1738 were dissolved in saline with $5 \%$ dimethylsulfoxide and $5 \%$ polyethyleneglycol-(15)-hydroxystearate (Kolliphor HS15, Sigma-Aldrich). For intracerebral infusion, PNU282987 and SSR180711 were dissolved in Dulbecco phosphate-buffered saline (dPBS). All a7ligand solutions were made immediately before treatment.

\section{Animals and MAM administration}

MAM (20 mg/kg i.p.) or saline ( $1 \mathrm{ml} / \mathrm{kg}$ i.p.) was administered on GD17 to individually housed timed pregnant Sprague-Dawley rats. Male offspring ( 94 control and 89 MAM from 25 and 29 litters, respectively) were weaned on PND22 and housed in groups of 2-3 littermates prior to experiments. Food and water were available ad libitum; rats were housed at constant room temperature $\left(22^{\circ} \mathrm{C}\right)$ and humidity (47\%) under a $12 \mathrm{~h}$ light-dark cycle (lights off at 7:00 pm). Protocols were performed according to National Institute of Health Guide for the Care and Use of Laboratory Animals and were approved by the Institutional Animal Care and Use Committee of the University of Pittsburgh.

Treatments with a7 nAChR modulators

Systemic administration was done through the lateral tail vein (1.5 $\mathrm{ml} / \mathrm{kg}$ ). Controls received vehicle (saline, 5\% DMSO, 5\% polyethyleneglycol-(15)-hydroxystearate). Intracerebral administration was done through the slow infusion $(0.25 \mu \mathrm{L} / \mathrm{min}$ during $2 \mathrm{~min})$ of solutions into the ventral hippocampus (vHipp) (AP $=-6.0 \mathrm{~mm}$, $\mathrm{ML}= \pm 4.8 \mathrm{~mm}, \mathrm{VD}=+7.0 \mathrm{~mm}$ from skull) or basolateral amygdala (BLA) $(\mathrm{AP}=-2.8 \mathrm{~mm}, \mathrm{ML}= \pm 4.7 \mathrm{~mm}, \mathrm{VD}=+7.6 \mathrm{~mm}$ from skull) bilaterally. Control groups were infused with vehicle (dPBS $0.5 \mu \mathrm{L})$. Treatments were randomly assigned in each litter. All doses were selected based on literature (Table 1) and calculated as free base.

VTA DA neuron recordings

Adult animals (PND65-140) were anesthetized with chloral hydrate $(400 \mathrm{mg} / \mathrm{kg}$ i.p. supplemented when necessary), placed in a stereotaxic and their body temperature was maintained at $37^{\circ} \mathrm{C}$ using a heating pad. For DA neuron recordings, single glass microelectrodes (6-8 $M \Omega$ ) filled with 2\% Chicago sky blue dissolved in $2 \mathrm{M} \mathrm{NaCl}$ were lowered in the VTA, starting at: AP $=-5.3 \mathrm{~mm}, \mathrm{ML}=-0.6 \mathrm{~mm}, \mathrm{VD}=+6.5 \mathrm{~mm}$. Spontaneously active neurons were recorded with open filter settings (low pass $=10 \mathrm{~Hz}$; high pass $=10 \mathrm{kHz}$ ).

To investigate the systemic effects of drugs, the electrode was slowly lowered into the VTA (maximum deep $=9.0 \mathrm{~mm}$ ) until a neuron was isolated that met the electrophysiological criteria of DA neurons based on location, firing rate and pattern and waveform [21]. Baseline activity was recorded for $60 \mathrm{~s}$. Immediately after, drugs were administered and activity was recorded for $120 \mathrm{~s}$. Firing rate $(\mathrm{Hz})$ and percentage of spikes fired in bursts were compared before and after drug administration. Burst initiation and termination were defined as previously described [22]. To investigate the effects after intracerebral administration, drug infusion was performed $15 \mathrm{~min}$ before population activity recording.

Population activity was determined by performing 6-9 consecutive vertical electrode tracks through the VTA separated by $200 \mu \mathrm{m}$ in a preset pattern. Spontaneous activity of each DA neuron was recorded for at least $1 \mathrm{~min}$. Three properties were analyzed: population activity (number of active DA neurons per electrode track), firing rate $(\mathrm{Hz})$ and percentage of action potentials occurring in bursts. The recording site was marked via electrophoretic ejection of Chicago sky blue $(-20 \mu \mathrm{A}$ for $20-30$ min). Animals were euthanatized by anesthetic overdose and decapitated. The brains were removed, fixed in $8 \%$ paraformadehyde in $0.2 \mathrm{M}$ PBS and cryoprotected in $25 \%$ sucrose in $0.2 \mathrm{M}$ PBS. Sixty-micrometer sections through the extent of the VTA, vHipp and BLA were stained with a mix of $95 \%$ Neutral Red and $5 \%$ Cresyl Violet for verification of electrode and cannula placements. To determine the medial-lateral distribution of the VTA DA neurons recorded, data were analyzed with respect to the electrode placement as previously described [23]. To be included in the analysis, data must be available from all three (medial, central and lateral) VTA locations.

Data analysis

Electrophysiological data of neuron firing properties were collected and analyzed using LabChart and NeuroExplorer software. Analyses of cell properties before and after drug administration were performed using paired Student's $t$ test and the significance levels were corrected for multiple comparisons using the Holm-Bonferroni method. Population activity analyses were done using two-way analysis of variance (ANOVA), with gestational treatment (GT: saline or MAM) as the first factor and acute treatment (AT: a7 ligands or vehicle) as the second factor, followed by Bonferroni post-hoc test. Statistics were calculated using Sigma Stat.

\section{RESULTS}

a7 $\mathrm{nAChR}$ agonists and PAMs did not impact the firing rate or pattern of VTA DA neurons

Initially, the impact of a7 nAChR modulators on the frequency and percentage of spikes in bursts of isolated DA neurons was tested. None of the drugs significantly altered the firing rate (control: Fig. 1a, Paired $t$ test, adjusted significance level $p^{\prime}>1.000$; MAM: Fig. 1c, Paired $t$ test, adjusted significance level $p^{\prime}>0.115$ ) or the proportion of spikes in bursts (control: Fig. $1 \mathrm{~b}$, Paired $t$ test, adjusted significance level $p^{\prime}>0.215$; MAM: Fig. $1 \mathrm{~d}$, Paired $t$ test, adjusted significance level $p^{\prime}>0.200$ ).

a7 nAChR agonists and type II PAM produce distinct effects on the number of spontaneously active VTA DA neurons in control vs. MAM rats

Drug treatment significantly affected the number of spontaneously active DA neurons (Fig. 2b, two-way ANOVA, GT: 


\section{SALINE}
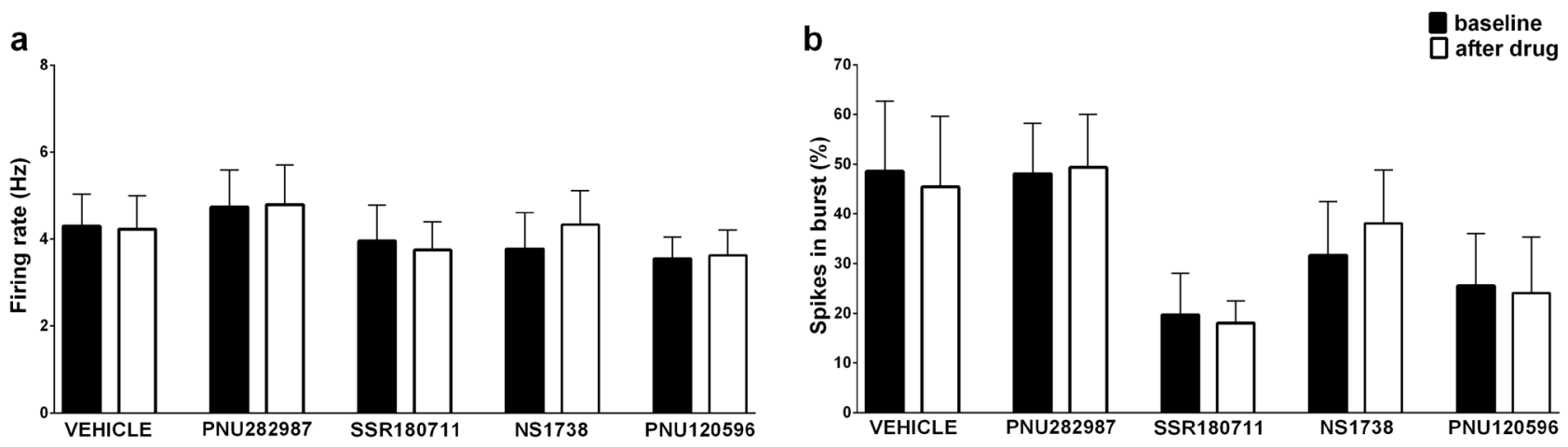

MAM
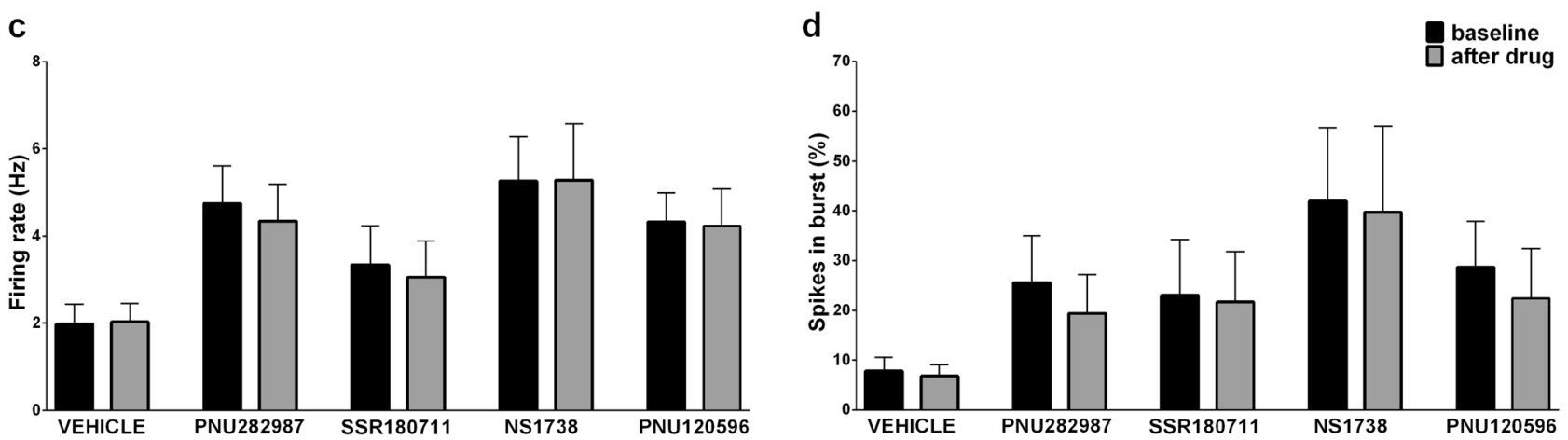

Fig. $1 \alpha 7 \mathrm{nAChR}$ agonists and PAMs did not change the firing rate or pattern of VTA DA neurons after acute i.v. administration ( $n=7-8$ cells/ group). a Mean firing rate $(\mathrm{Hz})$ and $\mathbf{b}$ percentage of spikes in bursts (\%) of the recorded DA neurons during baseline and after drug administration in control (saline) rats. $\mathbf{c}$ Mean firing rate $(\mathrm{Hz})$ and $\mathbf{d}$ percentage of spikes in bursts of the recorded DA neurons during baseline and after drug administration in MAM rats. Data are expressed as mean \pm S.E.M

$F_{(1,76)}=2.805, p=0.098$, AT: $F_{(4,76)}=6.472, p<0.001$, interactions: $\left.F_{(4,76)}=2.552, p=0.046\right)$ and their percentage of spikes in bursts (Fig. 2d, two-way ANOVA, GT: $F_{(1,693)}=0.0001, p=0.991$, AT: $F_{(4,693)}=1.810, p=0.125$, interactions: $\left.F_{(4,}, 693\right)=2.856$, $p=0.023)$. No significant impact of drug administration was found on DA neurons firing rate (Fig. 2c, two-way ANOVA, GT: $F_{(1}$, ${ }_{693)}=0.758, p=0.384$, AT: $F_{(4,693)}=3.023, p=0.017$, no difference detected by Bonferroni test, interactions: $F_{(4,693)}=2.310$, $p=0.056$ ).

As previously reported, MAM rats showed significantly higher numbers of spontaneously active DA neurons compared to controls (Fig. $2 b, 1.0 \pm 0.08$ cells/track for saline + vehicle vs. 1.7 \pm 0.11 cells/track for MAM + vehicle, Bonferroni test, $p<0.001$ ) and no differences in firing rate or percentage of spikes in burst (Fig. 2c,d, respectively). In control rats, PAM II PNU120596 administration impacted two measures: it increased the number of DA neurons recorded (1.7 \pm 0.25 cells/track, Fig. $2 b$, Bonferroni test, $p=0.012)$ and their bursting activity (37.1 $\pm 3.5 \%$, Fig. $2 \mathrm{~d}$, Bonferroni test, $p=0.002$ ). No significant effects induced by the other a7 nAChR modulators were detected in controls. The a7 $n A C h R$ ligands produced an opposite effect on VTA DA neuron population activity in MAM rats compared to controls. Both the full agonist PNU282987 and the partial agonist SSR180711 decreased the number of spontaneously active DA neurons (Fig. 2b, Bonferroni test, $p=0.012$ and $p=0.011$ for PNU282987 and SSR180711, respectively), reaching values similar to that found in the control group (Fig. 2b, Bonferroni test, $p>0.05 ; 1.0 \pm$ 0.14 cells/track for MAM + PNU282987 and $1.0 \pm 0.17$ cells/track for MAM + SSR180711). No significant changes in VTA DA neuron population activity in MAM rats were found following NS1738 or
PNU120596 (Fig. 2b) and none of the drugs changed the neurons bursting activity (Fig. 2d).

Intra-hippocampal infusion of a7 nAChR agonists produces similar effects as systemic administration

Since the $a 7 \mathrm{nAChR}$ agonists were active in MAM rats, the site of action of these agents was assessed. Given that the vHipp has a high density of a7 nAChR [24] and is the site driving the increase in VTA DA population activity in MAM rats [20], the impact of drug infusion into this structure was examined. Once more the MAM rats showed elevated DA neuron population activity $(1.7 \pm 0.14$ cells/track vs. $0.9 \pm 0.13$ cells/track for saline + vehicle group, Fig. 3b, two-way ANOVA, GT: $F_{(1,43)}=1.690, p=0.201$, AT: $F_{(2,43)}$ $=0.755, p=0.476$; interaction: $F_{(2,43)}=6.883, p=0.003$; Bonferroni test, $p<0.001$ ). Infusion of a7 $\mathrm{nAChR}$ agonists into the vHipp of MAM rats significantly decreased DA neuron population activity (Fig. 3b, Bonferroni test, PNU282987: $1.1 \pm 0.12$ cells/track, $p=$ 0.021; SSR180711: $1.0 \pm 0.10$ cells/track, $p=0.007$ ) without significantly impacting controls (Fig. $3 b$, Bonferroni test, $p>0.05$ ). MAM rats DA neurons showed a small but significant increase in average firing rate $(4.3 \pm 0.3 \mathrm{~Hz}$ vs. $3.5 \pm 0.3 \mathrm{~Hz}$ in controls, Fig. $3 c$, two-way ANOVA, GT: $F_{(1,342)}=1.218, p=0.271$, AT: $F_{(2,342)}=$ $1.716, p=0.181$; interaction: $\mathrm{F}_{(2,342)}=4.635, p=0.010$, Bonferroni test, $p=0.048)$ that was reversed by SSR $180711(3.1 \pm 0.2 \mathrm{~Hz}$, Fig. $3 c$, Bonferroni test, $p=0.008$ ). Two-way ANOVA showed a significant effect of PNU282987 treatment on burst firing (Fig. 3d, two-way ANOVA, GT: $F_{(1,342)}=0.575, p=0.449$, AT: $F_{(2,342)}=$ 3.904, $p=0.021$; interaction: $F_{(2,342)}=2.714, p=0.068$, Bonferroni test, $p=0.011$ vs. vehicle) revealing that this drug decreased the percentage of spikes in bursts in both MAM and control rats. 
a

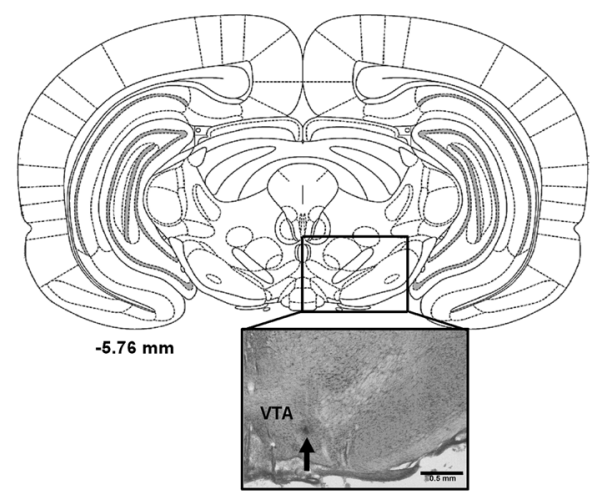

C

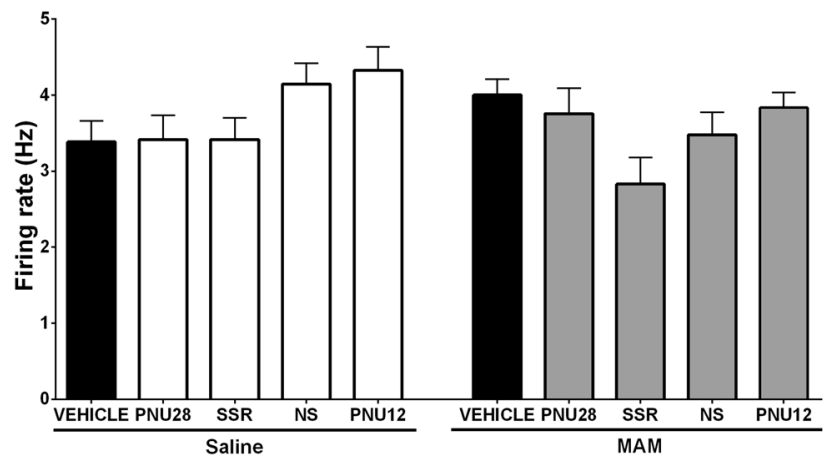

b

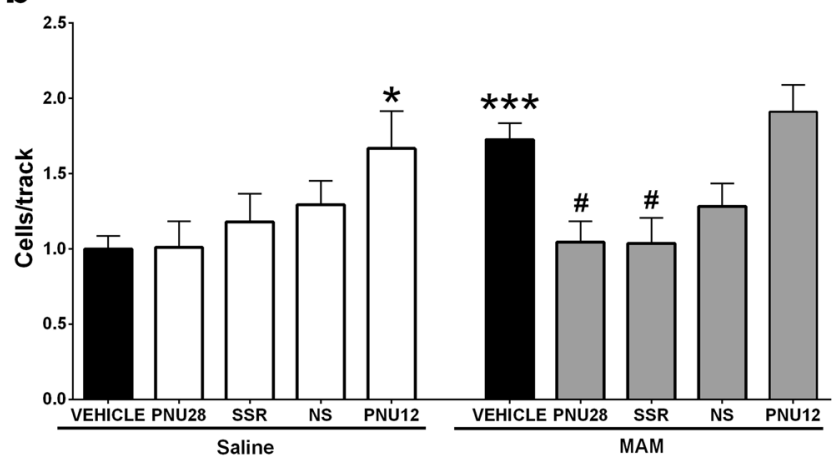

d

Fig. $2 \alpha 7$ nAChR agonists PNU282987 and SSR180711 decreased the number of spontaneously active VTA DA neurons in MAM rats while the PAM II PNU120596 increased population activity in control rats. a Histological representation of the electrode placement in the VTA. Arrow shows the blue dot resulting from Chicago sky blue electrophoretic ejection. Scale bar $=0.5 \mathrm{~mm}$. $\mathbf{b}$ Number of spontaneously active DA neurons found per electrode track in VTA $(n=8-11$ rats/group). c Mean firing rate $(\mathrm{Hz})$ and $\mathbf{d}$ percentage of spikes in bursts of the recorded DA neurons ( $n=50-105$ cells/group). Data are expressed as mean \pm S.E.M. Bonferroni post-hoc test: ${ }^{*} p<0.05,{ }^{* *} p<0.01,{ }^{* * *} p<0.001$ comparing with saline + vehicle, $p<0.05$ comparing with MAM + vehicle

Infusion of a7 nAChR agonists into the BLA increases VTA DA neuron population activity in control rats

Another structure with a high density of a7 nAChR [24] that impacts VTA DA neuron population activity is the BLA $[25,26]$. Both the a7 nAChR full agonist $(1.8 \pm 0.18$ cells/track $)$ and the partial agonist ( $1.6 \pm 0.19$ cells/track) infused into the BLA induced a significant increase in the number of DA neurons spontaneously active in the VTA of control rats $(1.0 \pm 0.13$ cells/track, Fig. $4 \mathrm{~b}$, twoway ANOVA, GT: $F_{(1,42)}=0.334, p=0.567, \mathrm{AT}: F_{(2,42)}=4.572$, $p=0.016$; interaction: $F_{(2,42)}=4.562, p=0.016$, Bonferroni test, $p$ $<0.001$ and $p=0.019$ for PNU282987 and SSR180711, respectively). In contrast, the increase in VTA DA neuron population activity in the MAM rats $(1.6 \pm 0.13$ cells/track, Fig. $4 b$, Bonferroni test, $p=0.008$ ) was not impacted by the drugs (Fig. $4 \mathrm{~b}$, Bonferroni test, $p>0.05$ ). An increase in burst firing of VTA DA neurons was observed in MAM rats infused with the partial agonist SSR180711 $(30.5 \pm 4.0 \%$ vs. $18.5 \pm 2.7 \%$ for MAM + vehicle group, Fig. 4 d, twoway ANOVA, GT: $F_{(1,403)}=0.617, p=0.433, \mathrm{AT}: F_{(2,403)}=1.454$, $p=0.235$; interaction: $F_{(2,403)}=3.605, p=0.028$, Bonferroni test, $p$ $=0.008$ ). Once more, the values for percentage of spikes in bursts in all experimental groups are in the normal range for VTA DA neurons, thus the physiological significance of this difference is unlikely. No statistically significant change was induced by treatments in DA neurons mean firing rate (Fig. 4c, two-way ANOVA, GT: $\left.F_{(1,403)}=1.289, p=0.257, \quad \mathrm{AT}: F_{(2,} 403\right)=0.668$, $p=0.513$; interaction: $\left.F_{(2,403)}=1.135, p=0.322\right)$.

VTA subregion-specific effects of a7 nAChR agonists and PAMs on DA neuron population activity

DA neurons are not homogenous across the VTA. The medial-lateral extent of this structure have distinct afferent inputs and projection targets. DA neurons located in the medial VTA project primarily to the ventromedial striatum (mesolimbic pathway), whereas laterally located VTA DA neurons project to more dorsal parts of the striatum (associative striatum - AST) [27]. As previously described [23], MAM rats exhibited elevated DA neuron activity across the medial-lateral extent of the VTA (Fig. 5). Taken together all the saline + vehicle and MAM + vehicle groups (independent of administration route) showed significant increases in the number of DA cells/track in MAM rats in both medial $(1.7 \pm 0.16$ cells/track vs. $1.0 \pm 0.09$ cells/track, Student's $t$ test, $t=3.914, p<0.001)$, central $(1.6 \pm 0.11$ cells/track vs. $0.9 \pm 0.10$ cells/track, Student's $t$ test, $t=4.583, p<0.001)$ and lateral regions of the VTA $(1.7 \pm 0.14$ cells/track vs. $0.9 \pm 0.11$ cells/track, Student's $t$ test, $t=4.279$, $p<0.001$ ).

Intravenous infusion of the agonists reduced the number of active DA neurons across the VTA of MAM rats (Fig. 5, top row); however, the effects are more robust in the lateral VTA, reaching statistical significance (Fig. 5c, two-way ANOVA, GT: $F_{(1,75)}=0.345$, $p=0.559 ;$ AT: $F_{(4,75)}=2.231, p=0.074$; interaction: $F_{(4,75)}=3.618$, $p=0.009$; Bonferroni test, $0.8 \pm 0.16$ cells/track, $p=0.009$ for PNU282987; $0.8 \pm 0.28$ cells/track, $p=0.009$ for SSR180711 vs. $1.9 \pm 0.24$ cells/track in MAM + vehicle group). Interestingly, the increase in DA neuron population activity induced by PNU120596 in normal rats seems to be more evident in the medial tracks $(2.1 \pm 0.39$ cells/track vs. $1.2 \pm 0.16$ cells/track in saline + vehicle group, Fig. 5a, two-way ANOVA, GT: $F_{(1,75)}=1.979, p=0.164$; AT: $F_{(4,75)}=3.880, p=0.006$, no difference detected by Bonferroni test; interaction: $\left.F_{(4,75)}=0.710, p=0.588\right)$. No significant changes were found in central VTA (Fig. 5b, two-way ANOVA, GT: $F_{(1,75)}=2.571, p=0.113 ;$ AT: $F_{(4,75)}=3.957, p=0.006$, no 

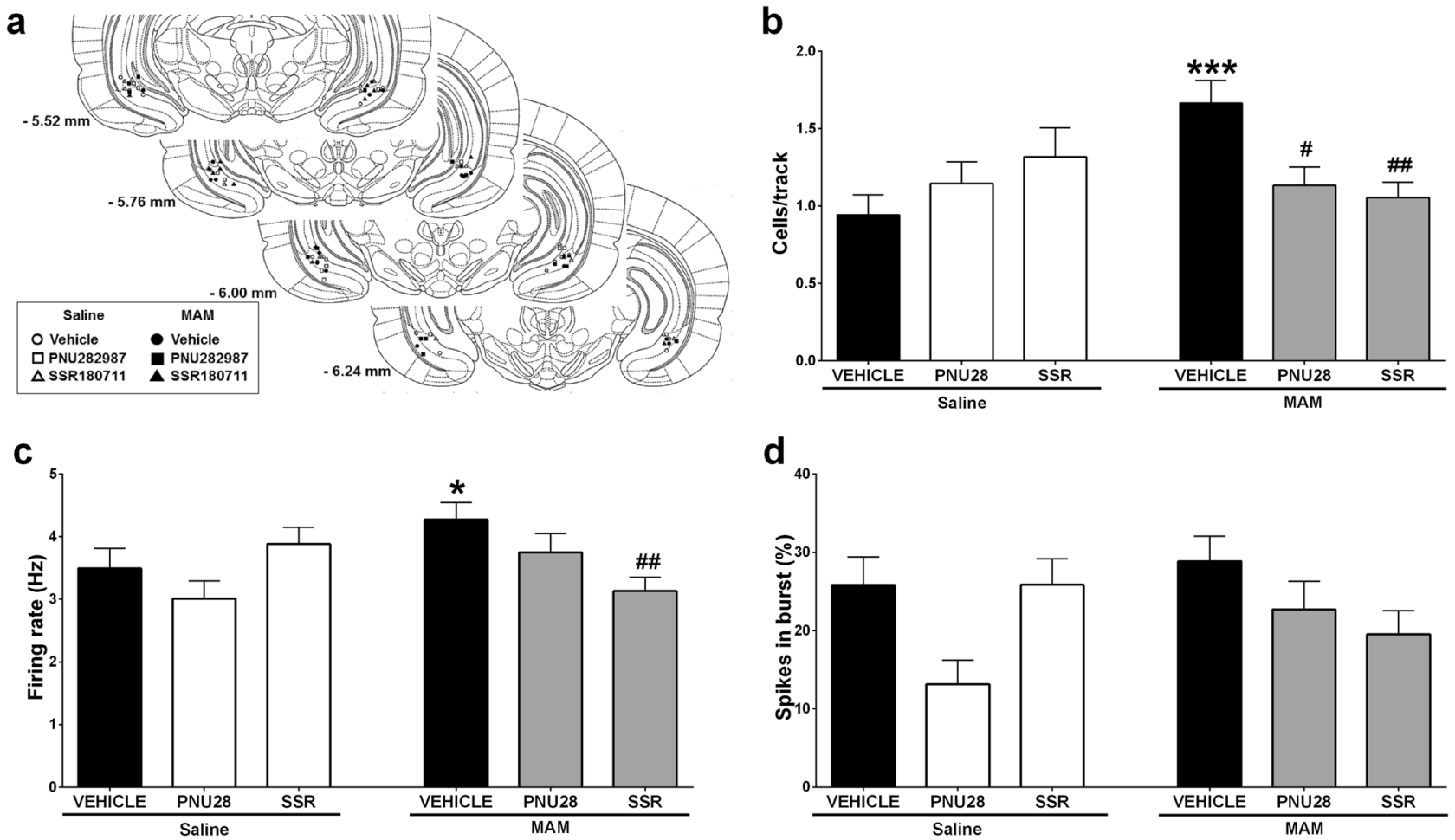

Fig. 3 Infusion of $\alpha 7$ nAChR agonists PNU282987 and SSR180711 into the vHipp reproduced the decrease in the number of spontaneously active DA neurons in the VTA of MAM rats observed after systemic administration. a Representation of the cannula placements in the vHipp. $\mathbf{b}$ Number of spontaneously active DA neurons recorded per electrode track in VTA ( $n=8-9$ rats/group). c Mean firing rate (Hz) and d percentage of spikes in bursts of the recorded DA neurons $(n=49-79$ cells/group). Data are expressed as mean \pm S.E.M. Bonferroni post-hoc test: ${ }^{*} p<0.05,{ }^{* * *} p<0.001$ comparing with saline + vehicle, ${ }^{\#} p<0.05,{ }^{\# \#} p<0.01$ comparing with MAM + vehicle

difference detected by Bonferroni test; interaction: $F_{(4,75)}=0.712$, $p=0.587)$.

Once more, the same pattern of effects produced by systemic administration of PNU282987 and SSR180711 in MAM rats was observed after intra-vHipp infusion. The reduction in the number of spontaneously active DA neurons was selectively influenced by changes in the lateral VTA (Fig. 5f, two-way ANOVA, GT: $F_{(1,43)}$ $=0.139, p=0.711$; AT: $F_{(4,43)}=4.831, p=0.013$; interaction: $F_{(4}$ $43)=4.831, \quad p=0.010$, Bonferroni test, $0.6 \pm 0.21$ cells $/$ track, $p<0.001$ for PNU282987; $0.6 \pm 0.16$ cells/track, $p=0.001$ for SSR 180711 vs. $1.7 \pm 0.22$ cells/track in MAM + vehicle group). No statistically significant differences in the medial (Fig. $5 \mathrm{~d}$, twoway ANOVA, GT: $F_{(1,43)}=2.087, p=0.156$; AT: $F_{(2,43)}=0.478$, $p=0.623$; interaction: $\left.F_{(2,43)}=1.932, p=0.157\right)$ or central VTA (Fig. 5e, two-way ANOVA, GT: $F_{(1,43)}=0.154, p=0.696$; AT: $F_{(2,43)}$ $=0.603, p=0.552$; interaction: $\left.F_{(2,43)}=2.073, p=0.138\right)$ were found.

After infusing a7 $\mathrm{nAChR}$ agonists into the $\mathrm{BLA}$, regional differences were also detected. Although the increase in DA neuron population activity can be observed across all regions of controls VTA, significant differences occurred exclusively in the central area (Fig. 5h, two-way ANOVA, GT: $F_{(1,41)}=0.0004$, $p=0.983$; AT: $F_{(2,41)}=5.956, p=0.005$; interaction: $F_{(2,41)}=7.688$, $p=0.001$; Bonferroni test, $2.1 \pm 0.23$ cells/track, $p<0.001$ for PNU282987; $1.8 \pm 0.22$ cells/track, $p<0.001$ for SSR180711 vs. $0.7 \pm 018$ cells/track in controls). No significant changes were detected in the other regions (Fig. $5 \mathrm{~g}$, medial VTA - two-way ANOVA, GT: $F_{(1,41)}=0.109, p=0.743 ;$ AT: $F_{(2,41)}=0.373, p=0.691$; interaction: $F_{(2,41)}=1.158, p=0.324$; Fig. 5i, lateral VTA - two-way ANOVA, GT: $F_{(1,41)}=0.0373$, $p=0.545 ;$ AT: $F_{(2,41)}=2.876, p=0.068$; interaction: $F_{(2,41)}=$ $0.679, p=0.513)$.

\section{DISCUSSION}

a7 nAChR agonists decrease VTA DA population activity in MAM rats via the vHipp

In our work, midbrain DA neurotransmission was affected by $a 7$ nAChR agonists and type II PAM by impacting the proportion of spontaneously active DA neurons in the VTA. Our data clearly showed that a7 nAChR agonists PNU282987 and SSR180711 can counteract the hyper-DA state of MAM rats with their likely site of action being the vHipp. This profile is consistent with the potential use of a7 nAChR agonists for schizophrenia treatment not only focusing on cognitive dysfunction, but also as a potential treatment for positive symptoms; and fits with the current search for drugs able to control DA function acting in structures upstream in the brain circuit. Dopamine dysfunction is a core feature in schizophrenia. Neuroimaging studies of schizophrenia patients showed the greatest increase in $\left[{ }^{18} \mathrm{~F}\right]$-DOPA uptake [28] and amphetamine-induced DA release [29] in the AST that correlates with exacerbation of psychosis. In humans, the dorsal tier of the substantia nigra projects to the AST; this is functionally equivalent to the lateral VTA projection to the core/lateral associative striatum in the rodent $[30,31]$. In the MAM model, the most significant increase in DA neuron population activity is in the lateral VTA, which is different from the change in population activity in the limbic medial VTA associated with amphetamine sensitization [23] and depression [32]. Therefore, the ability of the a7 nAChR agonists to normalize activity in the lateral VTA of the MAM model would be consistent with an antipsychotic effect. This selectivity also suggests that $a 7 n A C h R$ agonists may have less impact on aggravating negative symptoms of schizophrenia in comparison to antipsychotic drugs.

Our data showed that local infusion of the a7 nAChR agonists in the vHipp reduced VTA DA neuron population activity in MAM 


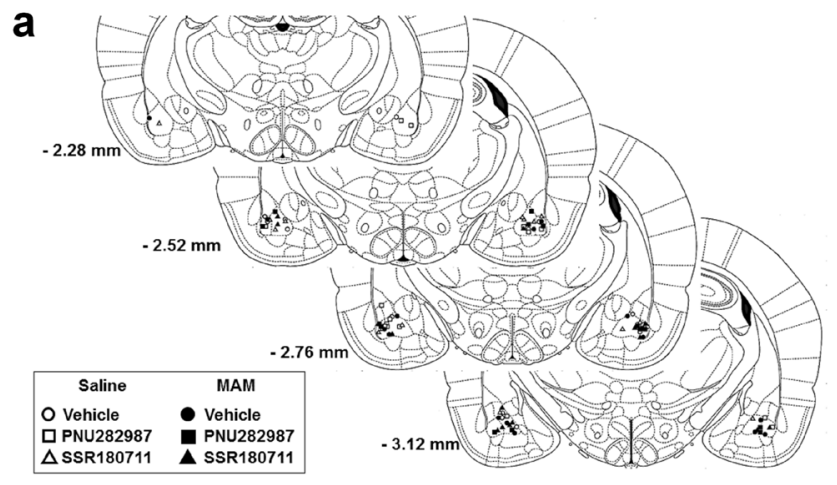

b
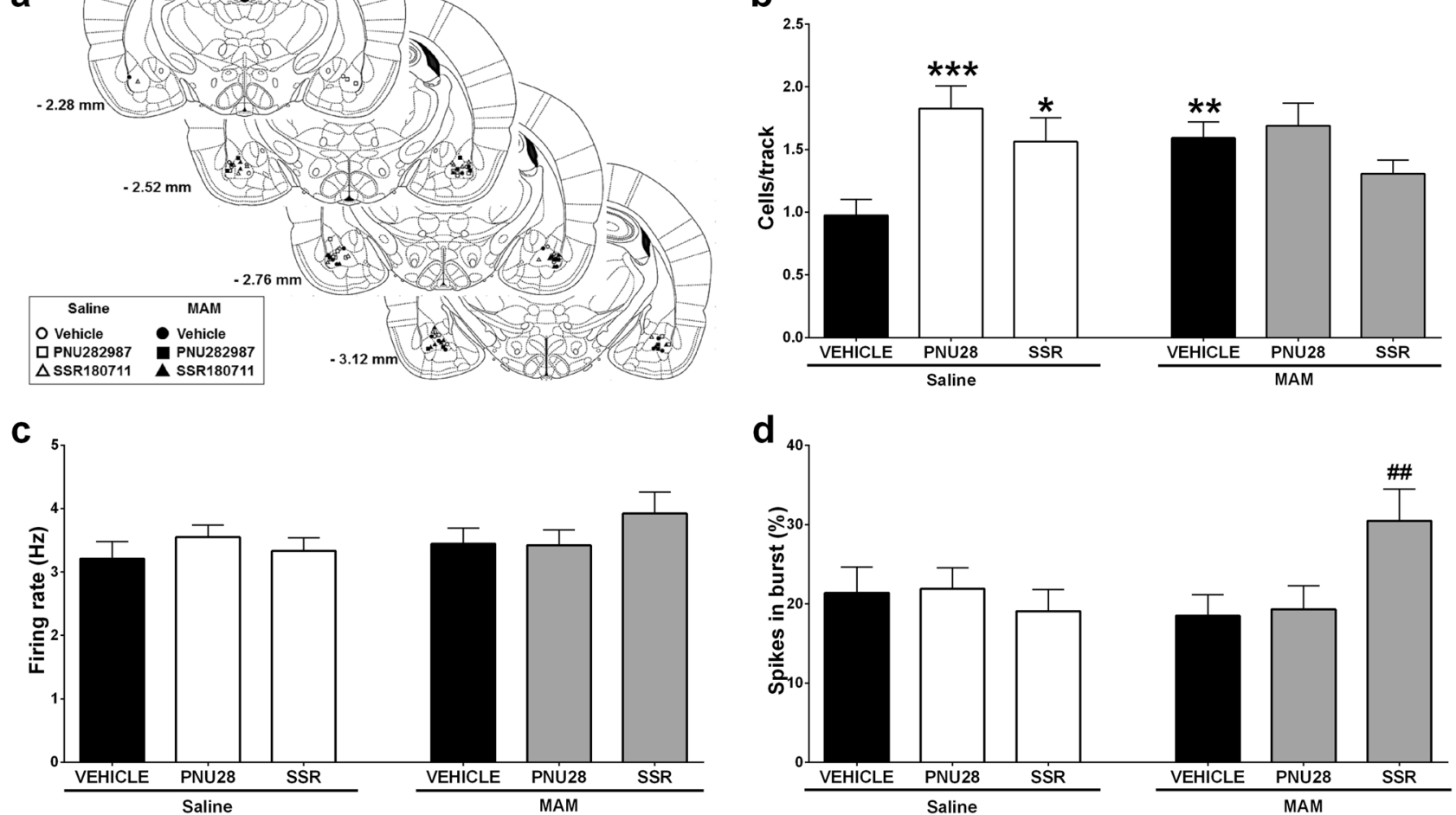

Fig. 4 Infusion of $\alpha 7$ nAChR agonists PNU282987 and SSR180711 into the BLA increased the number of spontaneously active DA neurons in the VTA of control rats without impacting MAM rats. a Histological representation of the cannula placements in the BLA. $\mathbf{b}$ Number of spontaneously active DA neurons found per electrode track in VTA ( $n=7-9$ rats/group). c Mean firing rate $(\mathrm{Hz})$ and $\mathbf{d}$ percentage of spikes in bursts (\%) of the recorded DA neurons ( $n=45-86$ cells/group). Data are expressed as mean \pm S.E.M. Bonferroni post-hoc test: ${ }^{*} p<0.05,{ }^{* *} p<$ $0.01,{ }^{* * *} p<0.001$ comparing with saline + vehicle, ${ }^{\# \#} p<0.01$ comparing with MAM + vehicle

rats, showing that activation/desensitization of a7 $\mathrm{nAChR}$ in this structure is sufficient to normalize VTA DA neuron activity in MAM rats. The rat hippocampus has a complex pattern of a7 nAChR expression; however, the majority of receptors are located in GABAergic interneurons [33, 34]. Activation of these receptors induces a long-lasting GABA release, increasing the frequency of inhibitory post-synaptic currents in pyramidal neurons [34, 35]. In this way, it is possible that potentiation of the activity of vHipp GABAergic interneurons could attenuate the hyperactivity of pyramidal neurons and, consequently, underlie the effects induced by the a7 nAChR agonists in MAM rats. In contrast, the lack of effect in controls is consistent with the fact that attenuation of vHipp activity in normal animals does not impact VTA DA neuron population activity $[20,36]$. Due to its widespread localization, a7 nAChR can potentiate both inhibitory and excitatory synapses in the hippocampus and, consequently, the net effect of drug administration can be dependent on the regional excitatory/inhibitory balance. Since there is not a disruption of the hippocampal excitatory/inhibitory balance in control animals, a7 nAChR agonist-induced modulation of hippocampal excitability may not be sufficient to trigger changes in VTA DA function through this pathway. Thus, the hippocampal hyperactivity likely makes MAM rats more sensitive to the effects of a7 nAChR agonists. Moreover, confirmation that a7 nAChR agonists can control hippocampal hyperactivity in MAM rats may further support their potential efficacy on cognitive disturbances.

However, changes in the cholinergic tone may also play a role in this differential sensitivity. Although there is little data regarding alterations in cholinergic transmission in the MAM GD17 model, increases in acetylcholine levels and ChAT activity were detected in the brain of MAM GD16.5 [37] and GD15 exposed rats [38] and a higher number of ChAT immunopositive cells in the medial septum was reported after GD12 exposure [39]. These data are consistent with the increase in acetylcholine and choline levels reported in schizophrenia patients [1]. While data regarding possible changes in the expression of a7 $n A C h R$ in the MAM model are absent, these alterations may impact dose dependency of the drugs without significantly impacting potential therapeutic actions.

a7 nAChR modulators increase VTA DA population activity in control rats: involvement of the BLA

In normal rats, we showed that only the a7 nAChR PAM II PNU120596 increased the number of spontaneously active DA neurons after systemic administration. The absence of effect of PNU282987 and NS1738 on DA population activity is consistent with previous DA microdialysis data, in which systemic administration of these drugs did not impact dopamine overflow in rats NAcc [40]. In contrast, infusion of a7 nAChR agonists in the BLA increased the number of active DA neurons in the VTA. The predominant effect of $B L A n A C h R$ on more medial/central regions of the VTA is in agreement with previous studies describing the increased cFos expression in the NAcc shell, but not in the NAcc core or in the dorsal striatum after systemic administration of both SSR180711 and PNU282987 [41, 42]. This profile is also consistent with previous data reporting BLA-mediated stress effects on VTA DA population activity [43], which would be consistent with a greater impact on affect or reward DA systems rather than psychosis [44].

It was recently proposed that the primary role of BLA a7 nAChR is to enhance inhibitory neurotransmission through activation of somatodendritic receptors on GABAergic interneurons [45]. Pharmacological activation of the BLA decreases the number of VTA DA neurons spontaneously firing; however, local infusion of TTX did not change the number of active VTA DA neurons [26]. Thus, a general inhibition of BLA activity induced by a7 nAChR 
Medial VTA

a

I.V.

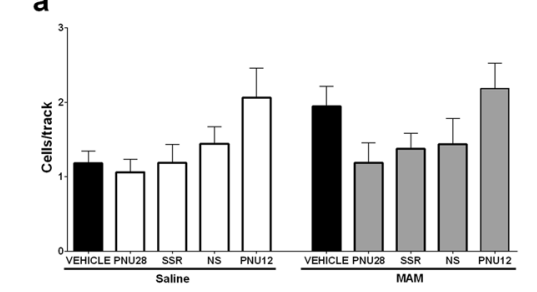

d
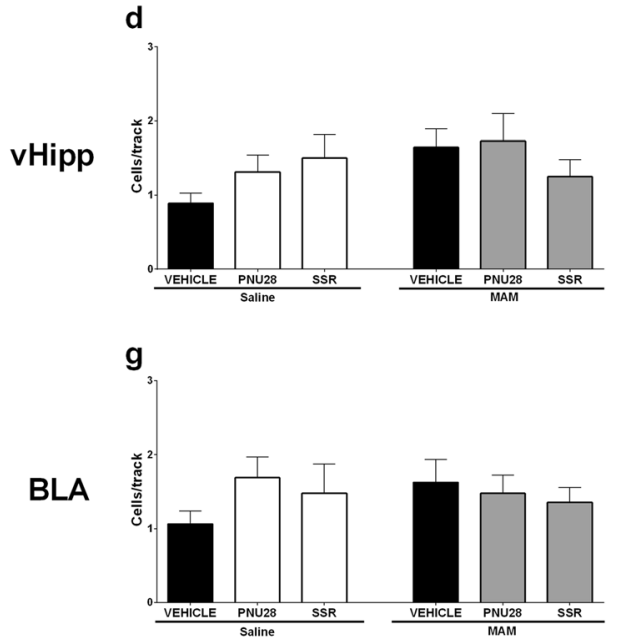

Central VTA

b

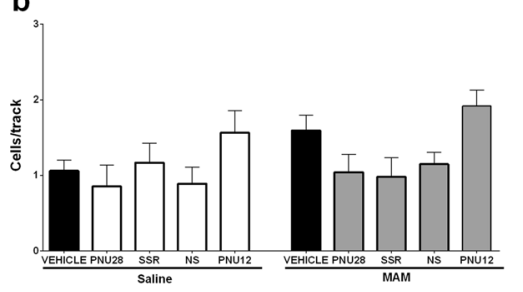

e

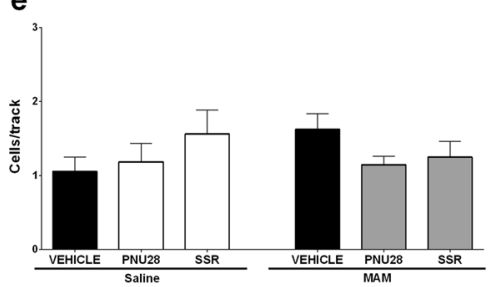

h

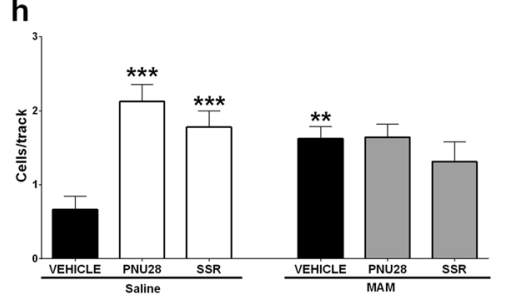

Lateral VTA

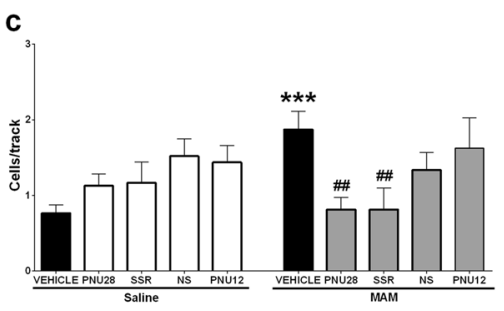

f

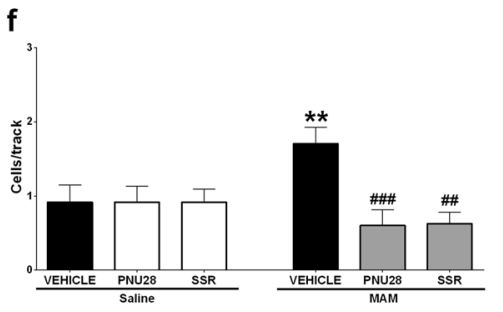

i

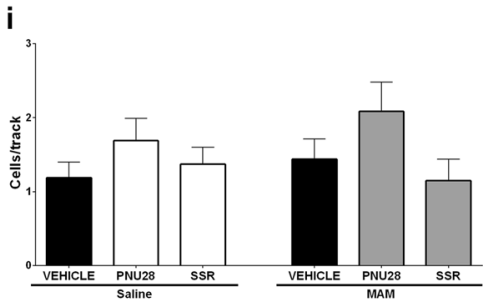

Fig. 5 Differential effects of the $\alpha 7$ nAChR agonists and PAMs on the number of spontaneously active DA neurons in control vs. MAM rats across the medial-lateral extent of the VTA. Number of spontaneously active DA neurons in the a medial $\mathbf{b}$ central $\mathbf{c}$ and lateral VTA after systemic (i.v.) injection of $\alpha 7 \mathrm{nAChR}$ modulators ( $n=8-11$ rats/group). Number of spontaneously active DA neurons in the $\mathbf{d}$ medial, e central and $f$ lateral VTA after vHipp infusion of $\alpha 7$ nAChR agonists ( $n=8-9$ rats/group). Number of spontaneously active DA neurons in the $\mathbf{g}$ medial, $\mathbf{h}$ central, and $\mathbf{i}$ lateral VTA after BLA infusion of $\alpha 7$ nAChR agonists $(n=7-9$ rats/group). Data are expressed as mean \pm S.E.M. Bonferroni post-hoc test: ${ }^{* *} p<0.01$, ${ }^{* *} p<0.001$ comparing with saline + vehicle, ${ }^{\# \#} p<0.01$, ${ }^{\# \#} p<0.001$ comparing with $\mathrm{MAM}+$ vehicle

agonists cannot explain the observed results. It is possible that a7 nAChR agonists may be acting on specific subsets of BLA neurons to activate VTA DA neurons. Such a selective effect may also account for the lack of change in VTA DA neuron activity observed in MAM rats. Since the BLA projects to the vHipp [46], it is possible that this BLA-vHipp projection participates in the observed effect, and that the already-present vHipp hyperactivation in the MAM rats obscures any additional activation by BLA afferents. Whether the $a 7 \mathrm{nAChR}$ can exert this projection-specific activation require further investigation.

\section{Final considerations}

In several cases, the a7 nAChR ligands impacted DA neuron firing rate and burst firing. While most of the changes were small in magnitude and may not represent a biologically significant effect, they support a differential a7 nAChR modulation of VTA DA neurons between MAM and control rats: increases in firing frequency and burst activity were mostly shown in control rats, whereas decreases in the same parameters were generally seen in MAM rats. Indeed, our data show that drug effects can vary according to the basal level of activity of specific brain circuits and highlights the importance of using appropriated animal models to make inferences about potential therapeutic use of new neuropsychiatric drug candidates.

We did not find any distinction with respect to functional impact across the drugs tested that could be specifically attributed to differences in their molecular mechanisms of action. Similar results have been reported by others after single drug administration [40, $47,48]$. Thus, it is likely that differences in the pharmacological profile among distinct a7 $\mathrm{nAChR}$ ligands will only be disclosed after repeated treatment. Although little tolerance has been seen regarding the procognitive effects of a7 nAChR ligands [42], the maintenance of the effect over DA transmission after repeated exposure has yet to be demonstrated and can elucidate further differences in their pharmacological profile.

Currently, few studies investigated the effects of a7 nAChR modulators in validated neurodevelopmental models of schizophrenia, and all of them focused on cognitive outcomes such as sensory processing, executive function, attention and memory [10, 18]. Beside ours, only two other studies employed the MAM model $[49,50]$ presenting conflicting results and with neither focusing on the DA system. While opposite effects of drug infusion into the vHipp versus the BLA were observed, our findings with systemic administration showed that, when the brain circuit is disrupted, the action of the a7 nAChR agonists in the vHipp appear to predominate. Finally, the apparent lack of effect of the type I PAM NS1738 in our experimental conditions should be interpreted with caution. Among the tested drugs, NS1738 has the shortest elimination half-life (42 $\mathrm{min})$ in rats [16] and its fast elimination may contribute to data variability.

In summary, a7 nAChR agonists and PAMs impact the DA system of rats in a state-dependent manner. The PAM II PNU120596 increased DA activity in normal rats whereas a7 $\mathrm{nAChR}$ agonists reversed the increase in DA neuron activity in the MAM model, and this effect appears to be mediated by their action in the vHipp. Moreover, local BLA infusion of the same agonists increases VTA DA activity in normal rats. Taking together, these data point to $a 7 \mathrm{nAChR}$ agonism as a promising strategy for 
the development of new pharmacological therapies for schizophrenia.

\section{ACKNOWLEDGEMENTS}

The authors thank Nicole MacMurdo and Christy Smolak for technical assistance.

\section{FUNDING}

This work was supported by grants from Conselho Nacional de Desenvolvimento Científico e Tecnológico (CNPq 200606/2015-8 - Brazil to GAN) and National Institute of Health (NIH MH57440 to AAG). AAG has received funds from Lundbeck, Pfizer, Otsuka, Lilly, Roche, Asubio, Abbott, Autofony, Janssen, Alkermes, Newron, Takeda. GAN declares no conflict of interest.

\section{ADDITIONAL INFORMATION}

Conflict of interest: The authors declare that they have no conflict of interest.

Publisher's note: Springer Nature remains neutral with regard to jurisdictional claims in published maps and institutional affiliations.

\section{REFERENCES}

1. Bustillo JR, Rowland LM, Lauriello J, Petropoulos H, Hammond R, Hart B, et al. High choline concentrations in the caudate nucleus in antipsychotic-naive patients with schizophrenia. Am J Psychiatry. 2002;159:130-3.

2. Holt DJ, Bachus SE, Hyde TM, Wittie M, Herman MM, Vangel M, et al. Reduced density of cholinergic interneurons in the ventral striatum in schizophrenia: an in situ hybridization study. Biol Psychiatry. 2005;58:408-16.

3. de Leon J, Diaz FJ. A meta-analysis of worldwide studies demonstrates an association between schizophrenia and tobacco smoking behaviors. Schizophr Res. 2005;76:135-57.

4. Rowe AR, Mercer L, Casetti V, Sendt KV, Giaroli G, Shergill SS, et al. Dementia praecox redux: a systematic review of the nicotinic receptor as a target for cognitive symptoms of schizophrenia. J Psychopharmacol. 2015;29:197-211.

5. D'Souza MS, Markou A. Schizophrenia and tobacco smoking comorbidity: $n A C h R$ agonists in the treatment of schizophrenia-associated cognitive deficits. Neuropharmacology. 2012;62:1564-73.

6. Castro NG, Albuquerque EX. Brief-lifetime, fast-inactivating ion channels account for the alpha-bungarotoxin-sensitive nicotinic response in hippocampal neurons. Neurosci Lett. 1993;164:137-40.

7. Castro NG, Albuquerque EX. Alpha-bungarotoxin-sensitive hippocampal nicotinic receptor channel has a high calcium permeability. Biophys J. 1995;68:516-24.

8. Freedman R, Wetmore C, Strömberg I, Leonard S, Olson L. Alpha-bungarotoxin binding to hippocampal interneurons: immunocytochemical characterization and effects on growth factor expression. J Neurosci. 1993;13:1965-75.

9. Jones IW, Wonnacott S. Precise localization of alpha7 nicotinic acetylcholine receptors on glutamatergic axon terminals in the rat ventral tegmental area. J Neurosci. 2004;24:11244-52.

10. Parikh V, Kutlu MG, Gould TJ. nAChR dysfunction as a common substrate for schizophrenia and comorbid nicotine addiction: current trends and perspectives. Schizophr Res. 2016;171:1-15.

11. Young JW, Geyer MA. Evaluating the role of the alpha-7 nicotinic acetylcholine receptor in the pathophysiology and treatment of schizophrenia. Biochem Pharmacol. 2013;86:1122-32.

12. Martin-Ruiz CM, Haroutunian VH, Long $P$, Young AH, Davis $K L$, Perry EK, et al. Dementia rating and nicotinic receptor expression in the prefrontal cortex in schizophrenia. Biol Psychiatry. 2003;54:1222-33.

13. Marutle A, Zhang $X$, Court J, Piggott $M$, Johnson $M$, Perry $R$, et al. Laminar distribution of nicotinic receptor subtypes in cortical regions in schizophrenia. J Chem Neuroanat. 2001;22:115-26.

14. Dempster EL, Toulopoulou T, McDonald C, Bramon E, Walshe M, Wickham H, et al. Episodic memory performance predicted by the $2 \mathrm{bp}$ deletion in exon 6 of the "alpha 7-like" nicotinic receptor subunit gene. Am J Psychiatry. 2006;163:1832-4.

15. Leonard S, Gault J, Hopkins J, Logel J, Vianzon R, Short M, et al. Association of promoter variants in the alpha7 nicotinic acetylcholine receptor subunit gene with an inhibitory deficit found in schizophrenia. Arch Gen Psychiatry. 2002;59:1085-96.

16. Timmermann DB, Grønlien JH, Kohlhaas KL, Nielsen EØ, Dam E, Jørgensen TD, et al. An allosteric modulator of the alpha7 nicotinic acetylcholine receptor possessing cognition-enhancing properties in vivo. J Pharmacol Exp Ther. 2007:323:294-307.
17. Hurst RS, Hajós M, Raggenbass M, Wall TM, Higdon NR, Lacwson JA, et al. A novel positive allosteric modulator of the alpha7 neuronal nicotinic acetylcholine receptor: in vitro and in vivo characterization. J Neurosci. 2005;25:4396-405.

18. Jones CK, Byun N, Bubser M. Muscarinic and nicotinic acetylcholine receptor agonists and allosteric modulators for the treatment of schizophrenia. Neuropsychopharmacol Rev. 2012;37:16-42.

19. Modinos G, Allen P, Grace AA, McGuire P. Translating the MAM model of psychosis to humans. Trends Neurosci. 2015;38:129-38.

20. Lodge DJ, Grace AA. Aberrant hippocampal activity underlies the dopamine dysregulation in an animal model of schizophrenia. J Neurosci. 2007:27:11424-30.

21. Grace AA, Bunney BS. Intracellular and extracellular electrophysiology of nigral dopaminergic neurons-1. Identification and characterization. Neuroscience. 1983;10:301-15.

22. Grace AA, Bunney BS. The control of firing pattern in nigral dopamine neurons: burst firing. J Neurosci. 1984;4:2877-90.

23. Lodge DJ, Grace AA. Divergent activation of ventromedial and ventrolateral dopamine systems in animal models of amphetamine sensitization and schizophrenia. Int J Neuropsychopharmacol. 2012;15:69-76.

24. Tribollet E, Bertrand D, Marguerat A, Raggenbass M. Comparative distribution of nicotinic receptor subtypes during development, adulthood and aging: an autoradiographic study in the rat brain. Neuroscience. 2004;124:405-20.

25. Du Y, Grace AA. Amygdala hyperactivity in MAM model of schizophrenia is normalized by peripubertal diazepam administration. Neuropsychopharmacology. 2016;41:2455-62.

26. Chang C, Grace AA. Amygdala-ventral pallidum pathway decreases dopamine activity following chronic mild stress in rats. Biol Psychiatry. 2014;76:223-30.

27. Ikemoto S. Dopamine reward circuitry: two projection systems from the ventral midbrain to the nucleus accumbens-olfactory tubercle complex. Brain Res Rev. 2007;56:27-78.

28. Howes OD, Montgomery AJ, Asselin MC, Murray RM, Valli I, Tabraham P, et al. Elevated striatal dopamine function linked to prodromal signs of schizophrenia. Arch Gen Psychiatry. 2009;66:13-20.

29. Kegeles LS, Slifstein M, Xu X, Urban N, Thompson JL, Moadel T, et al. Striatal and extrastriatal dopamine D2/D3 receptors in schizophrenia evaluated with [18F] fallypride positron emission tomography. Biol Psychiatry. 2010;68:634-41.

30. Grace AA, Gomes FV (2018). The circuitry of dopamine system regulation and its disruption in schizophrenia: insights into treatment and prevention. Schizophr Bull. 2018. https://doi.org/10.1093/schbul/sbx199

31. Chuhma N, Mingote S, Kalmbach A, Yetnikoff L, Rayport S. Heterogeneity in dopamine neuron synaptic actions across the striatum and its relevance for schizophrenia. Biol Psychiatry. 2017;81:43-51.

32. Moreines JL, Owrutsky ZL, Grace AA. Involvement of infralimbic prefrontal cortex but not lateral habenula in dopamine attenuation after chronic mild stress. Neuropsychopharmacoogyl. 2017;42:904-13.

33. Fabian-Fine R, Skehel P, Errington ML, Davies HA, Sher E, Stewart MG, et al. J Neurosci. 2001;21:7993-8003.

34. Yakel JL, Shao Z. Functional and molecular characterization of neuronal nicotinic ACh receptors in rat hippocampal interneurons. Prog Brain Res. 2004;145:95-107.

35. Hajos M, Hurst RS, Hoffmann WE, Krause M, Wall TM, Higdon NR, et al. The selective alpha7 nicotinic acetylcholine receptor agonist PNU-282987 [N-[(3R)-1Azabicyclo[2.2.2] oct-3-yl]-4-chlorobenzamide hydrochloride] enhances GABAergic synaptic activity in brain slices and restores auditory gating deficits in anesthetized rats. J Pharmacol ExpTher. 2005;312:1213-22.

36. Lodge DJ, Grace AA. The hippocampus modulates dopamine neuron responsivity by regulating the intensity of phasic neuron activation. Neuropsychopharmacology. 2006:31:1356-61.

37. Virgili M, Barnabei O, Contestabile A. Regional- and age-specific neurochemical alterations in rats rendered microencephalic by differentially timed gestational methylazoxymethanol treatment. Eur J Neurosci. 1989;1:647-53.

38. Johnston MV, Coyle JT. Ontogeny of neurochemical markers for noradrenergic, GABAergic, and cholinergic neurons in neocortex lesioned with methylazoxymethanol acetate. J Neurochem. 1980;34:1429-41.

39. Fiore M, Korf J, Angelucci F, Talamini L, Aloe L. Prenatal exposure to methylazoxymethanol acetate in the rat alters neurotrophin levels and behavior: considerations for neurodevelopmental diseases. Physiol Behav. 2000;71:57-67.

40. Marcus MM, Björkholm C, Malmerfelt A, Möller A, Påhlsson N, KonradssonGeuken $\AA$, et al. Alpha7 nicotinic acetylcholine receptor agonists and PAMs as adjunctive treatment in schizophrenia. An experimental study. Eur Neuropsychopharmacol. 2016;26:1401-11.

41. Hansen HH, Timmermann DB, Peters D, Walters C, Damaj MI, Mikkelsen JD. Alpha7 nicotinic acetylcholine receptor agonists selectively activate limbic regions of the rat forebrain: an effect similar to antipsychotics. J Neurosci Res. 2007;85:1810-8. 
42. Thomsen MS, Hay-Schmidt A, Hansen HH, Mikkelsen JD. Distinct neural pathways mediate a7 nicotinic acetylcholine receptor-dependent activation of the forebrain. Cereb Cortex. 2010;20:2092-102.

43. Valenti O, Gill KM, Grace AA. Different stressors produce excitation or inhibition of mesolimbic dopamine neuron activity: response alteration by stress pre-exposure. Eur J Neurosci. 2012;35:1312-21.

44. Grace AA. Dysregulation of the dopamine system in the pathophysiology of schizophrenia and depression. Nat Rev Neurosci. 2016;17:524-32.

45. Pidoplichko VI, Prager EM, Aroniadou-Anderjaska V, Braga MF. a7-Containing nicotinic acetylcholine receptors on interneurons of the basolateral amygdala and their role in the regulation of the network excitability. J Neurophysiol. 2013;110:2358-69.

46. Pikkarainen $M$, Rönkkö S, Savander V, Insausti R, Pitkänen A. Projections from the lateral, basal, and accessory basal nuclei of the amygdala to the hippocampal formation in rat. J Comp Neurol. 1999;403:229-60.

47. Nikiforuk A, Kos T, Hołuj M, Potasiewicz A, Popik P. Positive allosteric modulators of alpha 7 nicotinic acetylcholine receptors reverse ketamine-induced schizophrenia-like deficits in rats. Neuropharmacol. 2016;101:389-400.

48. Potasiewicz A, Nikiforuk A, Hołuj M, Popik P. Stimulation of nicotinic acetylcholine alpha7 receptors rescue schizophrenia-like cognitive impairments in rats. J Psychopharmacol. 2017;31:260-71.
49. Kohlhaas KL, Robb HM, Roderwald VA, Rueter LE. Nicotinic modulation of auditory evoked potential electroencephalography in a rodent neurodevelopmental model of schizophrenia. Biochem Pharmacol. 2015;97:482-7.

50. Mar AC, Nilsson SRO, Gamallo-Lana B, Lei M, Dourado T, Alsiö J, et al. MAM-E17 rat model impairments on a novel continuous performance task: effects of potential cognitive enhancing drugs. Psychopharmacol (Berl). 2017;234:2837-57.

51. Bodnar AL, Cortes-Burgos LA, Cook KK, Dinh DM, Groppi VE, Hajos M, et al. Discovery and structure-activity relationship of quinuclidine benzamides as agonists of alpha7 nicotinic acetylcholine receptors. J Med Chem. 2005;48:905-8.

52. Chan WK, Wong PT, Sheu FS. Frontal cortical alpha7 and alpha4beta2 nicotinic acetylcholine receptors in working and reference memory. Neuropharmacology. 2007;52:1641-9.

53. Pichat $P$, Bergis OE, Terranova JP, Urani A, Duarte C, Santucci V, et al. SSR180711, a novel selective alpha7 nicotinic receptor partial agonist: (II) efficacy in experimental models predictive of activity against cognitive symptoms of schizophrenia. Neuropsychopharmacology. 2007;32:17-34.

54. Bortz DB, Mikkelsen JD, Bruno JP. Localized infusions of the partial alpha 7 nicotinic receptor agonist SSR180711 evoke rapid and transient increases in prefrontal glutamate release. Neuroscience. 2013;255:55-67. 